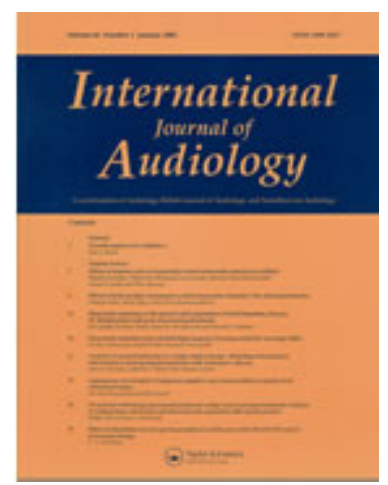

\title{
Readability Assessment of Self-Report Hyperacusis Questionnaires
}

\begin{tabular}{|r|l|}
\hline Journal: & International Journal of Audiology \\
\hline Manuscript ID & TIJA-2019-02-0075.R1 \\
\hline Danuscript Type: & Original Paper \\
\hline Author: & $\mathrm{n} / \mathrm{a}$ \\
\hline & $\begin{array}{r}\text { Complete List of Authors: } \\
\text { Sereda, Magdalena; University of Nottingham, Hearing Sciences, School } \\
\text { Of Medicine; NIHR Nottingham Biomedical Research Centre, Hearing } \\
\text { Sciences } \\
\text { Baguley, David; University of Nottingham, Hearing Sciences, Division of } \\
\text { Clinical Neuroscience ; National Institutes for Health Research } \\
\text { Nottingham, Hearing Sciences; Nottingham University Hospitals NHS } \\
\text { Trust, Nottingham Audiology Services }\end{array}$ \\
\hline Keywords: & Readability, Health literacy, Hyperacusis, Self-report questionnaires \\
\hline &
\end{tabular}


Abstract

Objective: To assess the overall readability of five currently available hyperacusis questionnaires and to assess the variability in readability of single items within each questionnaire. Design: Comparative study of five self-report hyperacusis questionnaires: (1) Geräuschüberempfindlichkeits-Fragebogen (GUF), (2) Noise Avoidance Questionnaire (NAQ), (3) Hyperacusis Questionnaire (HQ), (4) Sound Sensitive-Tinnitus Index (SSTI), and (5) Inventory of Hyperacusis Symptoms (IHS). Using well established readability formulas Flesh-Kincaid Grade Level (FKGL), Flesch Reading Ease (FRE), Simple Measure of Gobbledygook (SMOG) and FORCAST, in a computerised readability calculation software. Results: Reading levels calculated by each formula varied for every questionnaire. Readability scores ranged from 7.7 th to 12.7 th grade for overall readability depending on the questionnaire. This was higher than grade reading levels of 5-6th grade (10-12 years old) as recommended by the American Medical Association or 7-8th grade (12-14 years old) as recommended by the US National Institutes of Health. Single item readability analysis based on FKGL revealed that $32 \%$ to $70 \%$ of single items were written above the recommended grade levels. Conclusion: All five questionnaires are written at close to or exceeding the recommended grade levels. This requires attention from developers but also when interpreting the questionnaire scores obtained in clinic.

\section{Introduction}

Hyperacusis is an emergent diagnosis and a growing field of interest in the both the clinical and research communities. It is characterised as the 'perception of everyday environmental sounds as being overwhelmingly loud or intense' (Fackrell et al. 2017). Commonly co-incident with tinnitus, hyperacusis has often been assessed and measured using tinnitus-specific questionnaires. Understanding and knowledge of the condition has improved and now there are hyperacusis specific tools for diagnostic and measurement purposes. However, they are limited in number and varying degrees of robustness and psychometric validation (Fackrell and Hoare 2018). In brief, the questionnaires are concerned 
gathering examples of when and how hyperacusis affects the individual and to what extent, by grading the severity of hyperacusis based on the patient's answers.

Questionnaires are a fundamental part of clinical practice, especially when concerned with subjective, self-reported symptoms and conditions, such as hyperacusis. They allow for a time-efficient and structured assessment of symptoms and experience as well as facilitating the assessment of changes over time. Patient-reported outcome measures including questionnaires are an important part of patient-centred care, as they facilitate a deeper understanding of the effects of a condition on a patient's life (Douglas and Kelly-Campbell 2018). In order to gather complex information about the effects of a particular condition on one's life, it is important that the intended audience can easily read and understand the questionnaire. This introduces the concept of readability.

The readability of a particular text is the objective measure of the reading skills the person should have in order to be able to understand it (Badarudeen and Sabharwal 2010).This is quantified as the number of years of education equivalent to a reading grade level in the US grade system but can also be converted for other country specific systems, e.g. the key stage system in the UK. Commonly used formula are the Flesch Reading Ease (FRE) and Flesch-Kincaid Grade Level (FKGL) (Flesch 1948). The Simple Measure of Gobbledygook (SMOG; (McLaughlin 1969) is recommended for use with healthcare materials as it is based on more recent criteria for determining reading grade level, and has been reported to be the most suited and practical for application to health care materials (Wang et al. 2013). These formulas take into consideration number of syllables per word and or average words per sentence and are typically meant for use with prose-like text. This is problematic as questionnaires are rarely written in prose-like form, and are more typical to have a disjointed or stem and leaf format. The FORCAST formula (Caylor et al. 1973) is deemed to be most suited to assessing readability of text not in prose-like form such as 
Margol-Gromada, Readability of Hyperacusis Questionnaires

questionnaires, forms, lists test and job materials (Atcherson et al. 2013). It does not count number of sentences, or their average length but rather counts the number of monosyllabic words.

Single item analysis is another area of difficulty, as currently there are no widely used and validated readability formulas developed for single-item analysis specifically. Calderon and Morales et al. (2006) applied the FRE and FKGL to single item analysis of Quality of Life Questionnaires after combining stem-leaf format questions to form full sentences, in order to comply with recommendations to only assess running text (Flesch 1979). This method was also used by Betschart, Abt et al. (2018), and appears to be successful in overcoming the methodological challenge of assessing single items not in prose-like form.

Readability is an integral part of health literacy, definitions of which vary in the literature, however a systematic review by Sørensen et al. (2012) arrived at the following definition after thematic analysis of 17 eligible publications:

'Health literacy is linked to literacy and entails people's knowledge, motivation and competences to access, understand, appraise, and apply health information in order to make judgments and take decisions in everyday life concerning healthcare, disease prevention and health promotion to maintain or improve quality of life during the life course.'

Despite the importance of health literacy it is commonly neglected, a concerning issue given that health literacy is the single best predictor of an individual's health status (Badarudeen and Sabharwal 2010). In the US over $\$ 230$ billion a year is linked to low adult literacy, with nearly $50 \%$ of Americans finding understanding and using health information difficult (ProLiteracy 2019). In the UK, current health information is written at a level too complex for $43 \%$ of adults aged 16-65, if numeracy skills are required for comprehension of the information the figure rises to $61 \%$ (Rowlands et al. 2015). Ensuring that patients are able to fully understand matters pertaining to their health is simply a part of good practice, 
therefore adult health materials should be prepared at the lowest possible level of reading difficulty which is generally $5^{\text {th }}$ grade (Weiss and Coyne 1997). More specifically, important links between health literacy and rehabilitation outcomes for patients in the audiology clinic have been reported by (Gilligan and Weinstein 2014). The authors argue that in order for patient-centred care to succeed we need to ensure that the tools we use to enable the patient perspective within management options, are suitable i.e. readable by their target audience. If the readability of a questionnaire is too difficult this can lead to patients rejecting the questionnaire, providing partial information or answering in a way that does not truly reflect their experience (Atcherson et al. 2013). Therefore, reading grade levels of 5 to $6^{\text {th }}$ grade (1012 years old) are recommended by the American Medical Association (AMA) (Weiss 2007) and $7^{\text {th }}$ to $8^{\text {th }}$ grade (12-14 years old) are recommended by the U.S. National Institutes of Health (NIH) (Medlineplus 2017). Studies reporting on readability for tinnitus questionnaires (Atcherson, Zraick, and Brasseux 2011), Auditory Processing Disorder questionnaires (Atcherson et al. 2013), and Adult Audiology Rehabilitation outcome measures (Douglas and Kelly-Campbell 2018) indicate that readability of questionnaires and patient-reported outcome measures largely exceed the recommended reading levels mentioned above. These studies evaluated the overall readability of questionnaires, meaning that information about the variability in the readability of single items was not addressed. This is an important omission as very easy to read items can potentially skew the overall readability of the questionnaire towards lower grade, hiding the more difficult items; the resulting readability level would not reflect the true difficulty of the text (Homan, Hewitt, and Linder 1994; Betschart et al. 2018). Unlike running prose in which context can help the reader comprehend the meaning of a given text, respondents are required and expected to comprehend each item in a questionnaire separately (Calderón et al. 2006). Therefore, analysis of single items allows for a more comprehensive assessment of readability and can highlight particular items 
Margol-Gromada, Readability of Hyperacusis Questionnaires

within a questionnaire that require caution when interpreting patient's answers (Betschart et al. 2018).

The purpose of the present study is to assess the overall readability of five currently available hyperacusis questionnaires and to assess the variability in readability of single items within each questionnaire.

\section{Methods}

\section{Questionnaire selection}

Questionnaires were selected based on the following criteria: (i) focus on quantifying and characterising an individual's sound tolerance difficulties, (ii) questionnaire designed to be completed by the patient without help or guidance from a clinician. Questionnaires that were designed to be administered as a part of a semi-structured interview, or with clinician involvement were excluded. Whether the questionnaire was used in clinical practice or whether it undergone psychometric validation were not considered as exclusion criteria. Five questionnaires meeting the above criteria were selected for analysis:

- Geräuschüberempfindlichkeits-Fragebogen (GUF); English translation (Nelting and Finlayson 2004)

- Noise Avoidance Questionnaire (NAQ); English translation (Bläsing et al. 2010)

- Hyperacusis Questionnaire (HQ) (Khalfa et al. 2002)

- $\quad$ Sound Sensitive-Tinnitus Index (SSTI) (Greenberg 2017)

- Inventory of Hyperacusis Symptoms (IHS) (Greenberg and Carlos 2018) 
Margol-Gromada, Readability of Hyperacusis Questionnaires

\section{Formula selection}

There are a number of readability formula in existence; however no widely used formula that were specifically designed for single-item analysis. We selected the FRE, FKGL as they are most frequently utilised in readability literature facilitating comparison between the present study and published literature; the FORCAST as it is deemed the most suitable for readability analysis of questionnaires; and the SMOG formula has been reported to be most consistent, and most appropriate for application to healthcare material given it has an expected comprehension of $100 \%$ (Wang et al. 2013).

The FKGL, FORCAST and SMOG formulas give reading grade level scores, the higher the grade the more difficult the text it. The FRE is scored on a scale from 0-100 where a lower number is equivalent to a higher grade level score.

\section{Questionnaire format}

English versions of the questionnaires were first copied into a Word document, copying the exact format that was found in the source by MMG. MS then checked the copied versions for accuracy against the original source. To assess the overall readability of each questionnaire full sentences were formed from any preamble statements and question options; stem and leaf format questions were combined to form full sentences for each option. Question options that were short repeated words e.g. as with a 4 point scale 'never, sometimes, often, very often', are likely to score very easy and skew results, were removed from the analysis. This approach has been utilised by Calderon, Morales et al. (2006) and later by Betschart, Abt et al. (2018), based on recommendation from Flesch, that the FRE should only be used to test running text (Flesch 1979). All additional text including references, notes to the clinician were excluded from the analysis. 
Margol-Gromada, Readability of Hyperacusis Questionnaires

\section{Readability analysis}

Readability analysis was conducted using the software package Readability Studio Professional Edition version 2015 for Windows, (Oleander Software, Ltd, Vandalia, OH, USA). Descriptive statistics for mean, median, range and standard deviation were calculated using Microsoft Excel 2016 for Windows 10.

The readability analysis was conducted in three parts:

(1) Readability assessment of the questionnaires in their original format using the FORCAST formula.

(2) Readability assessment of the questionnaires where each item was manipulated to form full sentences, using the FRE, FKGL and SMOG formulas.

(3) Readability assessment of single items comprising each questionnaire, using the FRE and FKGL formulas, in keeping with the approach utilised by Calderon, Morales et al. (2006). 
Margol-Gromada, Readability of Hyperacusis Questionnaires

\section{Results}

Table one below details the factors that affect readability, and serves to illustrate the relative variability of each of the questionnaires. These statistics were extracted from the readability software, and can be used as a starting point for improving the readability of the questionnaires, by highlighting high numbers of complex words and sentence lengths, factors that increase the difficulty of a given text. Although it is important to note that the factors included in the table are not necessarily taken into consideration by the four formulas utilised in the study.

Table 1. Factors affecting readability for each questionnaire

\begin{tabular}{|c|c|c|c|c|c|c|}
\hline Questionnaire & $\begin{array}{l}\text { No. of } \\
\text { items }\end{array}$ & $\begin{array}{c}\text { Total } \\
\text { words }\end{array}$ & $\begin{array}{c}\text { Complex words } \\
\text { (3+syllables) }\end{array}$ & $\begin{array}{c}\text { Long words }(6+ \\
\text { characters })\end{array}$ & $\begin{array}{r}\text { Average } \\
\text { sentence } \\
\text { length } \\
\text { (words) }\end{array}$ & $\begin{array}{c}\text { Number of difficult sentences } \\
\text { (more than } 22 \text { words) }\end{array}$ \\
\hline$\overline{\text { GUF }}$ & 15 & 374 & $\begin{array}{c}21 \\
(5.6 \%)\end{array}$ & $\begin{array}{r}148 \\
(39.6 \%)\end{array}$ & 12.5 & 0 \\
\hline$\overline{\text { NAQ }}$ & 25 & 542 & $\begin{array}{r}54 \\
(10 \%) \\
\end{array}$ & $\begin{array}{r}166 \\
(30.6 \%) \\
\end{array}$ & 8.6 & 0 \\
\hline$\overline{\mathrm{HQ}}$ & 18 & 301 & $\begin{array}{r}40 \\
(13.3 \%)\end{array}$ & $\begin{array}{r}86 \\
(28.6)\end{array}$ & 6.4 & $\overline{0}$ \\
\hline$\overline{\text { SSTI }}$ & 20 & 712 & $\begin{array}{r}56 \\
(7.9 \%) \\
\end{array}$ & $\begin{array}{r}160 \\
(22.5 \%) \\
\end{array}$ & 17.7 & 3 \\
\hline IHS & 25 & 429 & $\begin{array}{r}31 \\
(7.2 \%) \\
\end{array}$ & $\begin{array}{r}115 \\
(26.8 \%) \\
\end{array}$ & 10.2 & 0 \\
\hline Median & 20 & 429 & 40 & 148 & 10.2 & $\overline{\overline{0}}$ \\
\hline Mean & 19.8 & 474 & 40.4 & 135 & 11.08 & 0.6 \\
\hline$\overline{\text { SD }}$ & 5.26 & 157.5 & 14.94 & 33.75 & 4.32 & 1.34 \\
\hline Range & $14-25$ & $\begin{array}{l}313- \\
712\end{array}$ & $21-56$ & $86-166$ & $6.4-17.7$ & $0-3$ \\
\hline
\end{tabular}

\section{Readability analysis using the FORCAST formula}

Readability analysis of the questionnaires in the original formats using the

FORCAST formula showed that the overall reading grade level for each of the 5

questionnaires exceeded the recommended reading level of 5- $6^{\text {th }}(10-12$ years old $)$ and $7^{\text {th }}$ to $8^{\text {th }}$ grade (12-14 years old), see table 2 . The software reports results according to the US grade system, which can be converted to country specific school years, in this case the UK year-group system, can be inferred by adding 1 year to the US grade results. 
Margol-Gromada, Readability of Hyperacusis Questionnaires

Table 2. FORCAST, FRE, FKGL and SMOG reading grade levels

\begin{tabular}{lcccc}
\hline Questionnaire & FORCAST & FRE & FKGL & SMOG \\
\hline GUF & 12.1 & 62 & 8.30 & 10 \\
\hline NAQ & 12.7 & 68 & 8.90 & 9.6 \\
\hline HQ & 9.9 & 63 & 7.80 & 10.8 \\
\hline SSTI & 8.9 & 58 & 10.1 & 12.7 \\
\hline IHS & 9.9 & 65 & 7.70 & 11.1 \\
\hline \hline median & 9.9 & 63 & 8.3 & 10.8 \\
\hline mean & 10.7 & 63.2 & 8.56 & 10.84 \\
\hline SD & 1.62 & 3.7 & 0.98 & 1.20 \\
\hline
\end{tabular}

Note that where a score has an integer after a decimal point e.g. 12.1 this means that the reading grade level is 12 th grade, 1 st month of academic year. FORCAST reading grade levels are for questionnaires in their original format. FRE, FKGL and SMOG scores are for the questionnaires in the manipulated full-sentence format. Conversion from FRE to grade level: 90-100- 5th grade; $80-90$ - 6th grade; 70 to $80-7$ th grade; $60-70-8$ th and 9th grade; $50-60-10$ th-12th grade; 30-50- college; 0 to 30- college graduate

\section{Readability analysis using the FRE, FKGL and SMOG formulas}

The grade levels as calculated by the FRE, FGKL and SMOG formulas for the questionnaires in the manipulated, full sentence format are shown in Table 2. There are some evident differences in the reading grade levels for each questionnaire depending on the readability formula used. With reference to the more conservative recommendations, all questionnaires exceed the grade 5-6 reading level. However, four out of the five questionnaires fall within the $7^{\text {th }}$ to $8^{\text {th }}$ grade level according to the FRE, and three out of five fall within the same criteria according to the FKGL. The SSTI has the highest reading grade levels as determined by each of the three formulas thus requiring a more advanced reading age than compared to the other questionnaires. Interestingly, SMOG reading grade levels for the IHS and the HQ exceed both the recommendations; however fall within the recommendations according to the FKGL formula. The differences in reading grade levels 
Margol-Gromada, Readability of Hyperacusis Questionnaires

could be attributed to differences between the formulas, although notably the SMOG formula yielded higher-grade reading levels for all questionnaires than compared to FRE and FKGL.

Figure 1 is a useful visualisation of how average words per sentence and average syllables per word map on to reading grade levels calculated by the FRE. The SSTI reading grade level as calculated by the FRE is equivalent to that of a $10-12^{\text {th }}$ grader (15-18 years old), as it has a high average number of words per sentence and average syllables per word. It is important to note that the FRE assumes that plain English has a score between 60-70, approximately equivalent to $7^{\text {th }}$ grade level, however the recommendations for healthcare materials is even lower.

Figure 1. Flesch Readability Chart for the five questionnaires showing the relation between average words per sentence, average syllables per word and the readability score yielded. 
Margol-Gromada, Readability of Hyperacusis Questionnaires

\section{Readability analysis of single items using FRE and FGKL formulas}

Single item analysis revealed variability in single item readability within each questionnaire (figure 2). Results show that a readability level above the maximum recommended $8^{\text {th }}$ grade level was found for $47 \%$ of items in the GUF; $44 \%$ of items in the NAQ; $39 \%$ of items in the HQ; $70 \%$ of items in the SSTI and $32 \%$ of items in the IHS. The highest score was a grade level of 16 according to the FKGL, and was found for single items within the NAQ, SSTI, IHS. According to the FRE, scores of 6, 15 and 27 were found for single items within the IHS, SSTI and NAQ respectively, meaning that a person would need the reading ability of a postgraduate to understand them.

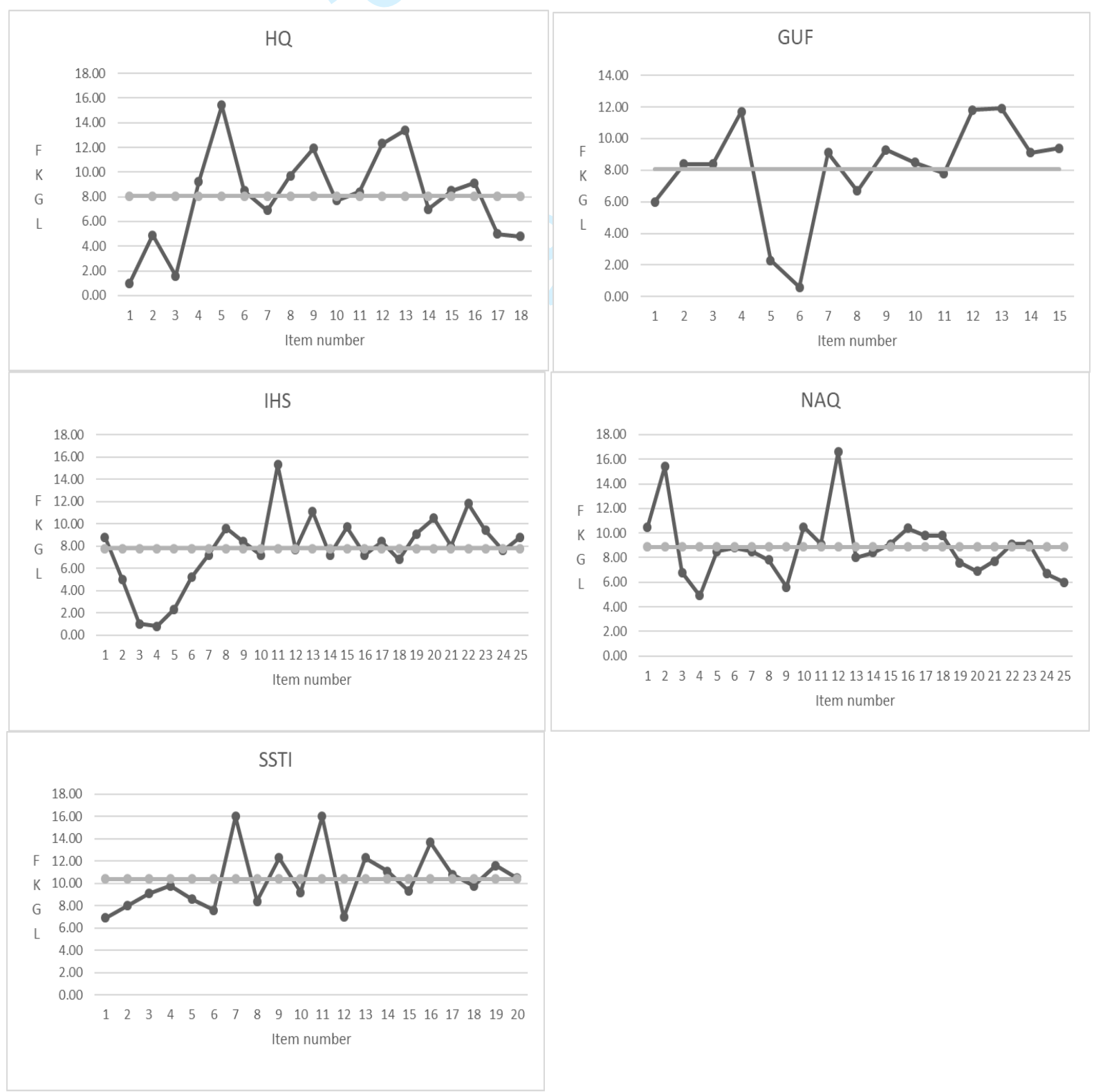


Margol-Gromada, Readability of Hyperacusis Questionnaires

Figure 2. Reading grade levels for single items of questionnaires. Solid light grey line represents the mean readability score for single items for each questionnaire.

\section{Discussion}

The present study assessed the readability of the currently available self-report hyperacusis questionnaires. Results revealed a range of reading grade levels for each questionnaire and across formulas, most of which clearly exceeded the recommended reading grade levels of $5-6^{\text {th }}$ grade and $7-8^{\text {th }}$ grade endorsed by the AMA and the NIH, respectively.

\section{Readability analysis using the FORCAST formula}

All questionnaires exceeded the grade reading level recommendations when the FORCAST formula was used, this is the only formula that is meant for text that is a nonprose format e.g. questionnaires. Similar to these findings are reports from Atcherson, Zraick and Brasseux (2011) who used FORCAST to assess tinnitus questionnaire readability. However, the expected comprehension for the FORCAST formula is only $35 \%$ and so it may not be the most appropriate formula to use for the healthcare setting. Primarily because ensuring that patients can comprehend $100 \%$ of the information they receive relating to their healthcare being an integral part of healthcare literacy and facilitating better healthcare outcomes for patients (Gilligan and Weinstein 2014; Douglas and Kelly-Campbell 2018). As patient reported outcome measures are utilised more clinical practice, research and used to inform healthcare services, it is crucial that patients are able to read and understand the questions (El-Daly et al. 2016). The NAQ yielded the highest grade reading level with the FORCAST of $12.7^{\text {th }}$ grade. Assuming the average reading age of 5-6 $6^{\text {th }}$ grade $(10-11$ years old) this would mean that around 5 million adults in England would not be able to read and comprehend the questionnaires (National Literacy Trust 2017). In the US this would translate to approximately 30 million adults that are classified as having a below basic health literacy 
Margol-Gromada, Readability of Hyperacusis Questionnaires

level not being able to comprehend the questionnaires (U.S. Department of Health and Human Services 2008).

\section{Readability analysis using the FRE, FGKL and SMOG formulas}

A common report in the readability literature is around the variability in the grade levels given by different formulas, which can be attributed to the parameters analysed by each formula (Atcherson et al. 2013). Similarly, in the present study, grade levels for a particular questionnaire varied approximately three grades depending on the formula (see table 2).

SMOG reading grade levels for every questionnaire clearly exceeded even the more lax recommendation of 7 to $8^{\text {th }}$ grade reading level, and whilst correlation analyses were not conducted, the SMOG and FKGL generally showed the same trend. Similar results have been reported for audiologic rehabilitation outcome measures by Douglas and Kelly-Campbell (2018), with the SMOG and FORCAST formulas yielding the highest reading grade level. The SMOG formula is not only recommended for use with healthcare materials but is based on more recent criteria for determining readability (Wang et al. 2013).

A difficulty that is not often discussed in the literature concerned with readability of questionnaires is that fact that other than the FORCAST, the other formulas should be applied to text in full sentences. Hence, why the manipulation of the questionnaires to achieve the most appropriate format for the formulas was carried out, yet it does highlight the strong need for a formula that can meet the specific requirements of $100 \%$ comprehension as well as being able to tackle non-prose like, stem-leaf formats as is common of questionnaires.

\section{Readability analysis of single items using the FRE and FGKL formulas}

Readability analysis of single-items allowed further insight into the readability issues within each questionnaire. The SSTI clearly exceeded the other four questionnaires, with 
$70 \%$ of items yielding a grade level above the maximum $8^{\text {th }}$ grade recommendation. This could affect the clinical usefulness of information gathered with these questionnaires, as patients with lower reading ages would be at risk of rejecting the questionnaires, or providing inaccurate information (Atcherson, Zraick, and Brasseux 2011). Whilst there are no guidelines on single item readability, reports in the literature suggest that the best way to facilitate meaningful, reliable and useful information gathering using questionnaires as a tool would be to ensure that every item is readable by patients (Gilligan and Weinstein 2014).

Readability is part of the wider concept of health literacy; poorer health literacy is associated with poorer healthcare outcomes. Douglas and Kelly-Campbell (2018) argue that ignoring basics such as the readability of self-report patient materials, such as questionnaires, can lead to patient's issues not being fully addressed for example if they have misunderstood questions or answered inaccurately and even leading to early discharge of the patient from the service. In research the issue may impact the accuracy of evaluating interventions, if the participant have not been able to understand a patient-report tool used as the outcome measure (Douglas and Kelly-Campbell 2018) essentially invalidating the empirical data collected (Atcherson, Zraick, and Brasseux 2011). Apart from potentially affecting the validity of the information gathered, the use of patient materials that are not suitable for even those with the lowest health literacy can create barriers to patients in accessing services they need (Rajah et al. 2018). There may be additional factors to be aware of, especially for patients with hyperacusis. It is common to have patients fill in questionnaires as they are waiting for appointments. Clinic waiting rooms are known to be busy and commonly noisy environments that can induce stress in patients even without hyperacusis, especially if noise levels exceed ambient noise level recommendations (Hill and LaVela 2015). A difficult soundscape coupled with a questionnaire that is written at a reading exceeding that of the patient, could introduce further difficulties for patients with hyperacusis. 
Margol-Gromada, Readability of Hyperacusis Questionnaires

Improving the readability of single items and therefore the overall readability should be a consideration for developers of questionnaires. Mono- or bi-syllabic word substitutions for unfamiliar medical words and reduction in length of sentences would help to improve readability (El-Daly et al. 2016). Edited versions of the questionnaires should undergo validation.

\section{Limitations}

A limitation of the present study, as with other reports, is that mother factors that affect readability such as format, text style and size and images (Atcherson, Zraick, and Brasseux 2011) were not analysed. Additionally, the lack of formulas appropriate for single item analysis required some manipulation of the questionnaires away from their original format. Only English versions of the questionnaires were analysed, so the reported results cannot be generalised to other translations that exist. Readability analysis on the original German language versions of the GUF and NAQ may be of clinical interest.

Despite the limitations, the present study presents important information on the readability of hyperacusis questionnaires, utilising format appropriate formulas and providing an insight into the single-item variability within some of the currently available questionnaires.

\section{Conclusion}

Researchers and developers should consider the overall readability of the developed questionnaires, ensuring that it is in keeping with recommendations. Furthermore, a greater awareness of and adherence to the recommendations should be made on the single item level, so that there is less variability within a questionnaire. Researchers and developers should also be aware of which questions may need some caution when interpreting patient answers, in the case that it has a very difficult readability. Further work on single item readability formula and methodological approaches including the creation of a computerised version of the 
Margol-Gromada, Readability of Hyperacusis Questionnaires

formula, to allow for more robust single item analysis would be a welcome addition to the research area.

Acknowledgements
Declaration of interest

No conflicts of interest to disclose. 
Margol-Gromada, Readability of Hyperacusis Questionnaires

References

Atcherson, Samuel R., Cynthia M. Richburg, Richard I. Zraick, and Cassandra M. George. 2013. "Readability of Questionnaires Assessing Listening Difficulties Associated with (Central) Auditory Processing Disorders." Language, Speech, and Hearing Services in Schools 44 (1):48-60. doi: 10.1044/0161-1461(2012/11-0055).

Atcherson, Samuel R., Richard I. Zraick, and Raven E. Brasseux. 2011. "Readability of Patient-Reported Outcome Questionnaires For Use With Persons With Tinnitus." Ear and Hearing 32 (5):671-3. doi: 10.1097/AUD.0b013e3182134654.

Badarudeen, Sameer, and Sanjeev Sabharwal. 2010. "Assessing readability of patient education materials: current role in orthopaedics." Clinical orthopaedics and related research 468 (10):2572-80. doi: 10.1007/s11999-010-1380-y.

Betschart, Patrick, Dominik Abt, Hans-Peter Schmid, Pascal Viktorin, Janine Langenauer, and Valentin Zumstein. 2018. "Readability assessment of commonly used urological questionnaires." Investigative and clinical urology 59 (5):297-304. doi: 10.4111/icu.2018.59.5.297.

Bläsing, Lena, Gerhard Goebel, Uta Flötzinger, Anke Berthold, and Birgit Kröner-Herwig. 2010. "Hypersensitivity to sound in tinnitus patients: An analysis of a construct based on questionnaire and audiological data." International Journal of Audiology 49 (7):518-26. doi: 10.3109/14992021003724996.

Calderón, José L., Leo S. Morales, Honghu Liu, and Ron D. Hays. 2006. "Variation in the readability of items within surveys." American journal of medical quality : the official journal of the American College of Medical Quality 21 (1):49-56. doi: $10.1177 / 1062860605283572$.

Caylor, J.S., T.G. Stitch, L.C. Fox, and J.P. Ford. 1973. "Development of a simple readability index for job reading material. ." In Paper presented at the meeting the annual meeting of the American Educational Research Association New Orleans, LA.

Douglas, A., and R. J. Kelly-Campbell. 2018. "Readability of patient-reported outcome measures in adult audiologic rehabilitation." American Journal of Audiology 27 (2):208-18. doi: 10.1044/2018_AJA-17-0095.

El-Daly, Ibraheim, Hajir Ibraheim, Karthig Rajakulendran, Paul Culpan, and Peter Bates. 2016. "Are patient-reported outcome measures in orthopaedics easily read by patients?" Clinical orthopaedics and related research 474 (1):246-55. doi: 10.1007/s11999-015-4595-0.

Fackrell, Kathryn , and Derek J. Hoare. 2018. "Scales and Questionnaires for Decreased Sound Tolerance " In Hyperacusis and Disorders of Sound Intolerance edited by Marc Fagelson and David M. Baguley, 43-58. San Diego Plural Publishing.

Fackrell, Kathryn, Iskra Potgieter, Giriraj S. Shekhawat, David M. Baguley, Magdalena Sereda, and Derek J. Hoare. 2017. "Clinical Interventions for Hyperacusis in Adults: A Scoping Review to Assess the Current Position and Determine Priorities for Research." BioMed research international 2017:2723715-. doi: 10.1155/2017/2723715.

Flesch, Rudolf 2018. "How to write plain English ", Accessed 10th October http://pages.stern.nyu.edu/ wstarbuc/Writing/Flesch.htm.

Flesch, Rudolph. 1948. "A new readability yardstick." Journal of Applied Psychology 32 (3):221-33. doi: 10.1037/h0057532.

Gilligan, Jennifer, and Barbara E Weinstein. 2014. "Health Literacy and Patient-Centered Care in Audiology - Implications for Adult Aural Rehabilitation." Journal of 
Communication Disorders, Deaf Studies \& Hearing Aids 2 (2):4. doi: 10.4172/jcdsha.1000110.

Greenberg, Ben. 2017. "Assessing the impact of sound sensitivity in tinnitus."

Greenberg, Benjamin, and Megan Carlos. 2018. "Psychometric Properties and Factor Structure of a New Scale to Measure Hyperacusis: Introducing the Inventory of Hyperacusis Symptoms." Ear and Hearing 39 (5):1025-34. doi: 10.1097/aud.0000000000000583.

Hill, Jennifer N., and Sherri L. LaVela. 2015. "Noise Levels in Patient Rooms and at Nursing Stations at Three VA Medical Centers." HERD: Health Environments Research \& Design Journal 9 (1):54-63. doi: 10.1177/1937586715592635.

Homan, Susan, Margaret Hewitt, and Jean Linder. 1994. "The Development and Validation of a Formula for Measuring Single-Sentence Test Item Readability." Journal of Educational Measurement 31 (4):349-58. doi: 10.1111/j.1745-3984.1994.tb00452.x.

Khalfa, S., S. Dubal, E. Veuillet, F. Perez-Diaz, R. Jouvent, and L. Collet. 2002. "Psychometric Normalization of a Hyperacusis Questionnaire." ORL 64 (6):436-42. doi: 10.1159/000067570.

McLaughlin, G. H. 1969. "SMOG grading: A new readability formula." Journal of Reading 12 (8):639-46.

Medlineplus. 2018. "How to Write Easy-to-Read Health Materials." U.S. National Library of Medicine, U.S. Department of Health and Human Services and National Institutes of Health, Accessed 11th November https://medlineplus.gov/etr.html.

National Literacy Trust. 2018. "Adult literacy." Accessed 22nd October https://literacytrust.org.uk/parents-and-families/adult-literacy/.

Nelting, M, and N.K. Finlayson. 2004. GÜF-Geräuschüberempfindlichkeits-Fragebogen. Göttingen: Hogrefe.

ProLiteracy. 2019. "Adult Literacy Facts." Accessed 2nd January https://proliteracy.org/Resources/Adult-Literacy-Facts.

Rajah, Retha, Mohamed Azmi Ahmad Hassali, Lim Ching Jou, and Muthu Kumar Murugiah. 2018. "The perspective of healthcare providers and patients on health literacy: a systematic review of the quantitative and qualitative studies." Perspectives in Public Health 138 (2):122-32. doi: 10.1177/1757913917733775.

Rowlands, Gillian, Joanne Protheroe, John Winkley, Marty Richardson, Paul T Seed, and Rima Rudd. 2015. "A mismatch between population health literacy and the complexity of health information: an observational study." British Journal of General Practice 65 (635):e379-e86. doi: 10.3399/bjgp15X685285.

Sørensen, Kristine, Stephan Van den Broucke, James Fullam, Gerardine Doyle, Jürgen Pelikan, Zofia Slonska, Helmut Brand, and Consortium Health Literacy Project European. 2012. "Health literacy and public health: A systematic review and integration of definitions and models." BMC Public Health 12 (1):80. doi: 10.1186/1471-2458-12-80.

U.S. Department of Health and Human Services. 2008. "Quick Guide to Health Literacy." In, edited by Office of Disease Prevention and Health Promotion. Washington, DCWashington, DC.

Wang, Lih-Wern, Michael J. Miller, Michael R. Schmitt, and Frances K. Wen. 2013. "Assessing readability formula differences with written health information materials: Application, results, and recommendations." Research in Social and Administrative Pharmacy 9 (5):503-16. doi: 10.1016/j.sapharm.2012.05.009. 
Margol-Gromada, Readability of Hyperacusis Questionnaires

Weiss, Barry, D. 2007. Health literacy and patient safety: Help patients understand. Manual for clinicians. . 2nd ed. Chicago American Medical Association and American Medical Foundation

Weiss, Barry, D., and Cathy Coyne. 1997. "Communicating with patients who cannot read." The New England Journal of Medicine 337 (4):272-4. 